\title{
The Features of Localized Plasticity Autowaves in Solids
}

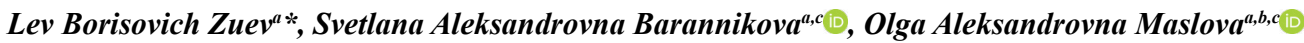 \\ ${ }^{a}$ Institute of Strength Physics and Materials Science, SB RAS, Tomsk, Russia \\ ${ }^{b}$ Universidade Federal de Minas Gerais, Belo Horizonte, Brasil \\ ${ }^{c}$ National Research Tomsk State University, Tomsk, Russia
}

Received: October 28, 2018; Revised: May 08, 2019; Accepted: May 21, 2019

The localized plastic deformation and the law-like regularities underlying its development in solids are considered. The characteristic features of localized plasticity are analyzed for a wide range of materials. Thus a correlation is established between the products of scales and of process rates obtained for the elastic and plastic deformation. It is a favorable ground for hypothesizing causal links between the elastic and plastic deformation by introducing an elastic-plastic invariant, which is the master equation of the autowave plasticity model being developed. Localized plasticity phenomena are proposed to be addressed in the frame of autowave and quasi-particle approach.

Keywords: Elasticity, plasticity, localization, crystal lattice, self-organization, autowave, propagation rate.

\section{Introduction}

During recent decades we have focused on the macroscale development of plastic flow. The results are presented in papers ${ }^{1-3}$ and in the monograph ${ }^{4}$. We have gradually come to accept that the plastic flow behavior has a characteristic attribute: the deforming medium would spontaneously separate into actively deforming layers alternating with inactive non-deformed layers (Fig. 1). As a result, an intricate arrangement of localized plas ticity patterns would emerge and vary in space and with time. The patterns have been correlated with the flow stages on the stress-strain curves, $\sigma(\varepsilon)$, plotted for the test samples. The available experimental evidence suggests that the patterns in question have space scales $\sim 10^{-2} \mathrm{~m}$ and characteristic times $\sim 10^{2} \ldots 10^{3} \mathrm{~s}$. The latter two values are virtually unaffected by the kind of material and only slight ly so, by the loading conditions.

The stratification of the plastically deforming medium (localization of deformation) is equivalent to the structure formation in the same; hence, this is actually related to the medium's self-orga niza tion ${ }^{5,6}$. Our understanding of this fact offers a clearer view of the nature of plasticity. Thus the author is responsible for much of the work on the patterns in question and for identifying them as 'localized plastic flow autowaves" . The autowave generation is known to involve a decrease in the entropy of the deforming medium ${ }^{8}$, which counts in favor of the self-organization concept. The autowave length, $\lambda$, and the time period, $T$, have been determined experimentally for similar processes; the values obtained are generally in the range $0.5 \cdot 10^{-2} \leq \lambda \leq 2 \cdot 10^{-2} \mathrm{~m}$ and $10^{2} \leq T \leq 10^{3}$ s. At about the same time, the theory of solitary plastic waves was put forward for an explanation of plastic flow ${ }^{9}$.
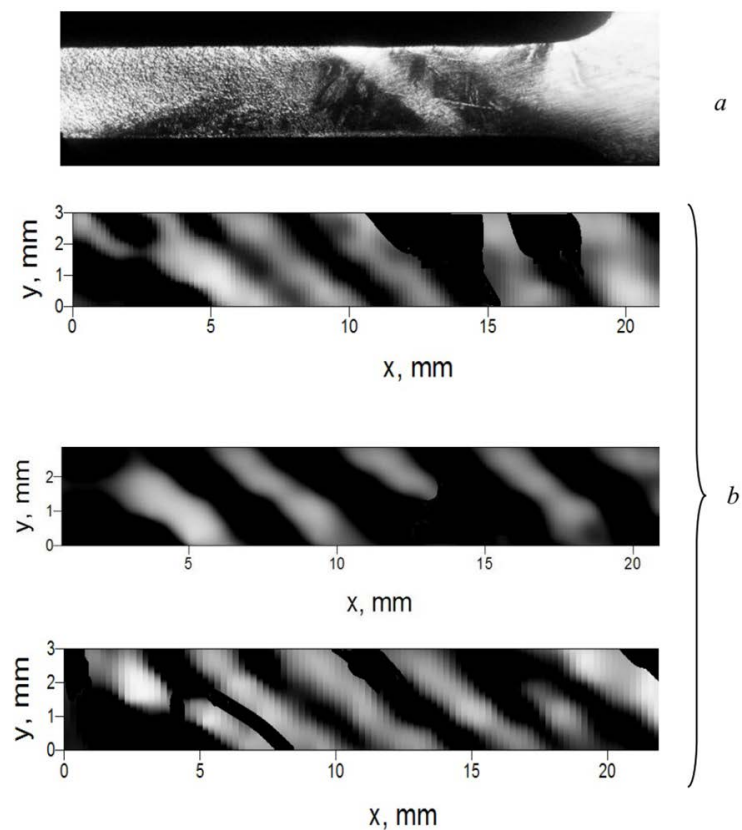

Figure 1. Plastic flow localization in the deformed single $\gamma$-Fe crystal (a) macrophotograph and (b) series of localized plasticity patterns observed for different times (dark and light bands correspond to active and passive material volumes, respectively)

Here reference should be made to the conceptual representation of other workers ${ }^{11,12}$, concerning the nature of periodic processes involved in the plastic deformation. Today the autowave model is substantiated by abundant theoretical and experimental evidence ${ }^{12-17}$. It is therefore claimed herein that the auto wave approach has presently received support among scientists. 


\section{Methods and Materials}

The experimental studies of autowave processes of plastic flow were done with the aid of double-exposure speckle photogra phy (Fig. 2 a); due to the stepwise stressing, the method was adapted to high plastic deformation ${ }^{4}$. The application of the method enables reconstruction of the displacement vector field for the flat sample, i.e. $|r(x, y)|>>\chi$ (here $\chi$ is the inter pla nar spacing). Using specially developed software, plastic distortion tensor components were calculated for different times and different points on the sample surface (Fig. 2 b). To obtain a generalizable set of data, the experiments were done for a variety of materials having dissimilar nature, which also differed in structure as well as in physical and mechanical properties. The range of studied materials included pure metals and alloys, alkali halide crystals and some rocks 3,4. The metals and alloys had FCC, BCC, HCP or tetragonal lattice; these were in single-crystal or polycrystalline state, polycrystalline metals and alloys differing in grain size.
We will discuss herein the general regularities of localized plasticity observed for all studied materials.

Autowave processes are likely to originate in the so-called active medium with energy sources distributed throughout its volume ${ }^{6,18,19}$. The elastic stress fields in the vicinity of stress concentrators play the role of energy sources; hence, the deforming medium meets the condition for autowave generation. An analysis of plastic flow should be based on space-time nonlinear kinetics relations derived for strains and stresses suitable for descriptions of response to loading of a nonlinear deforming medium. The derivation of such relations is considered herein. Moreover, a new universal approach to the phenomenon of solids plasticity is introduced in the form of logical implication. The aim of the given paper is an attempt at revealing the major regularities of the plastic flow by generalization of the diverse database, which would enable elaboration of a persuasive theory of this multivariate phenomenon.
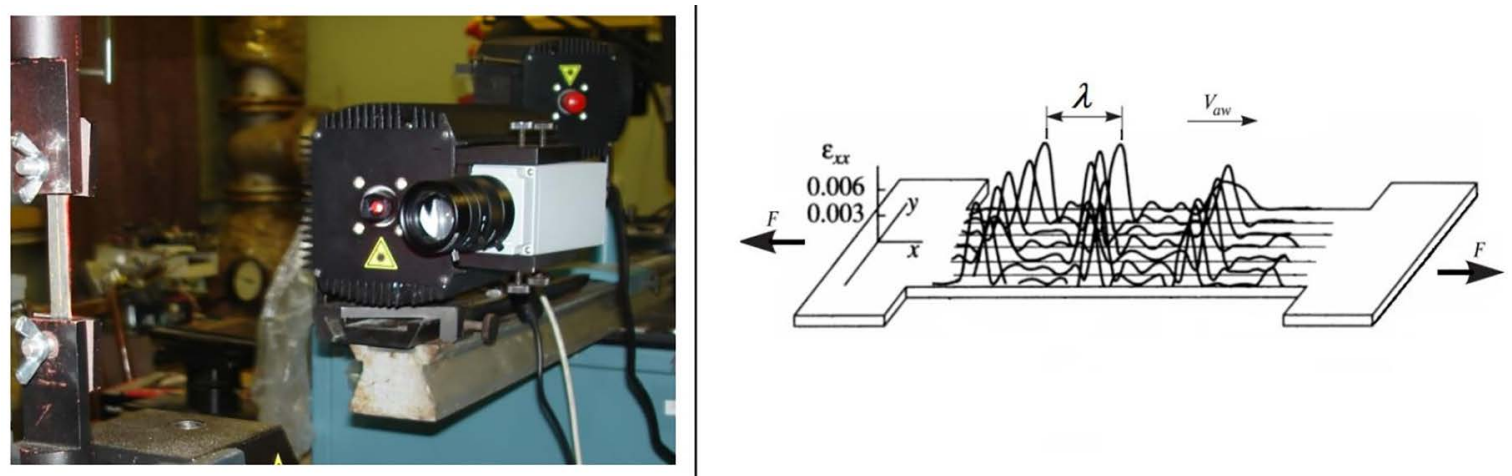

(a)

(b)

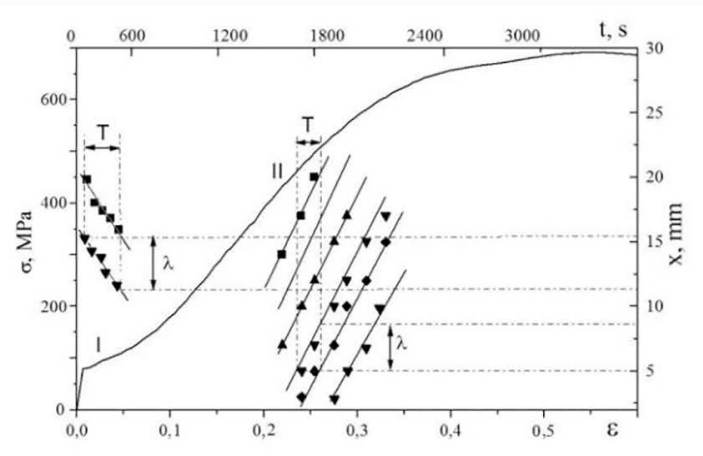

(c)

Figure 2. Localized plasticity in metals (a) - ALMEC-tv measuring system installed on an Instron-1185 testing machine; (b) - distribution of active and passive zones of strain localization; (c) - plastic flow curve and X -t diagram 


\section{The autowave nature of plastic deformation and the elastic-plastic invariant}

To gain an insight into the nature of localized plastic deformation, the regular features of its development have to be assessed on the base of qualitative data. The regularities exhibited by the localized plastic flow behavior have been determined with the maximal accuracy for the easy glide and linear work hardening stages. In these cases, the localization patterns comprise a set of localized plasticity nuclei having space and time periods, i.e. autowave length, $\lambda$, and characteristic time, $T$, respectively (Fig. 2 C); hence, the autowave propagation rate, $10^{-5} \leq \mathrm{V}_{\mathrm{aw}}=\mathrm{d} \lambda / \mathrm{dT} \leq 10^{-4} \mathrm{~m} / \mathrm{s}$.
Next Fig. 3 a-c shows the experimentally obtained data characterizing the localized plastic flow autowaves.

\subsection{Distinctive features of localized plasticity autowaves}

It is found that the autowave rate depends on a dimensionless characteristic of the plastic flow process, i.e. $\theta=E^{-1} \cdot d \sigma / d \varepsilon$ (here $E$ is the elastic modulus). This dependence has the form

$$
V_{a w}=V_{0}+\Xi / \theta \sim \theta^{-1},
$$

where $V_{0}$ and $\Xi$ are constants, which differ for the easy glide and linear work hardening stages. (Fig. 3 a).

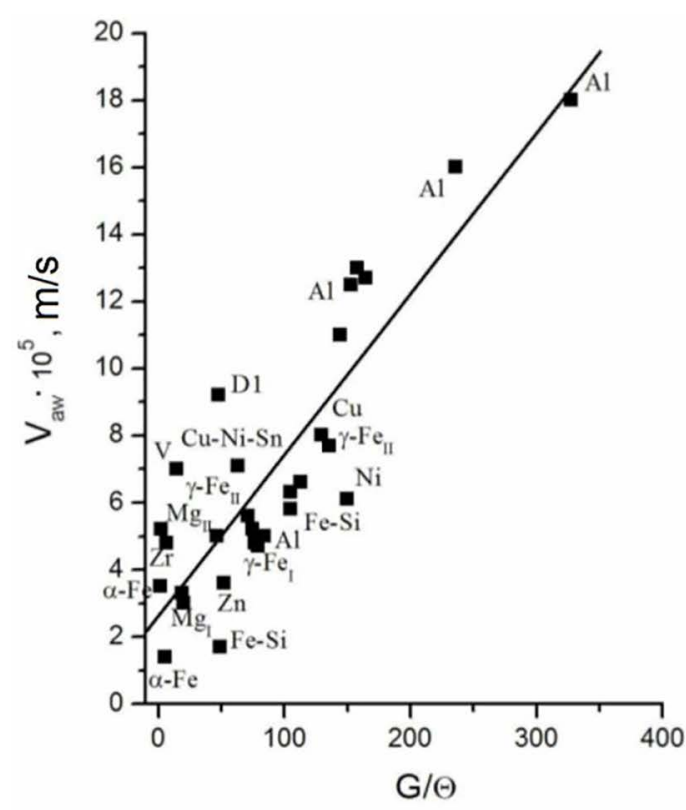

(a)

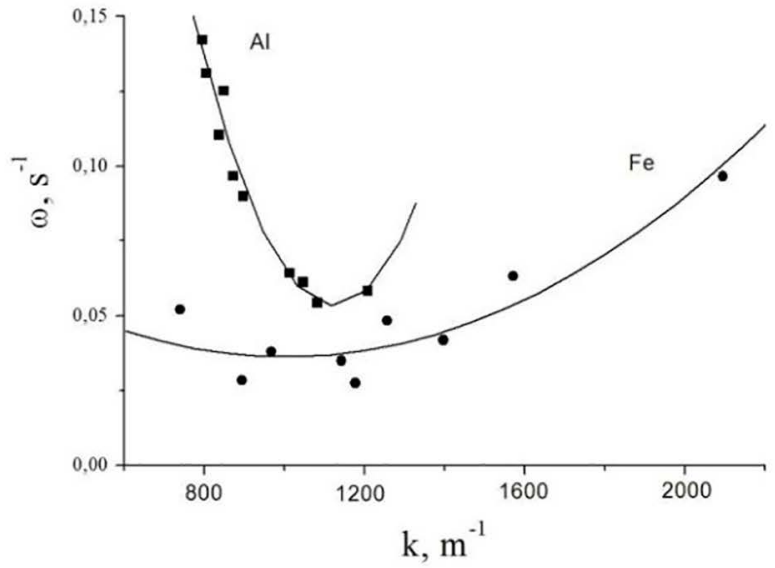

(b)

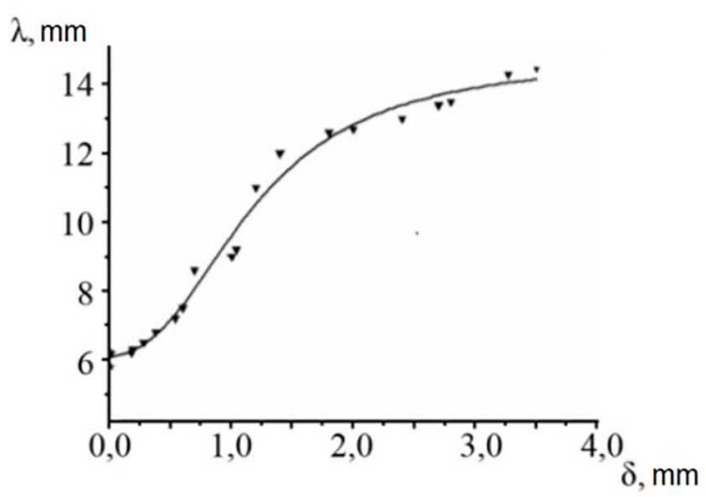

(c)

Figure 3. Distinctive features of localized plasticity autowaves (a) work hardening coefficient dependence of autowave rate; (b) dispersion relation; (c) grain size dependence of autowave length 
It should be emphasized that the autowaves observed for the latter two stages are described by the following quadratic dispersion relation (Fig. 3 b),

$$
\omega(k)=\omega_{0} \pm \alpha\left(k-k_{0}\right)^{2},
$$

where $\omega=2 \pi / T$ is frequency; $k=2 \pi / \lambda$ is wave number and $\omega_{0}, k_{0}$ and $\alpha$ are constants. The mi nus and plus signs correspond to the easy glide and linear work hardening stages, respectively. By substituting $\omega=\omega_{0} \cdot \tilde{\omega}$ and $k=k_{0} \pm \tilde{k} / \sqrt{\alpha / \omega_{0}}$ into Eq. (2), it is easily reduced to the form $\tilde{\omega}=1 \pm \tilde{k}^{2}$ (here $\tilde{\omega}$ and $\tilde{k}$ are dimensionless frequency and wave number, respectively).

The grain size dependence of autowave length, $\lambda(\delta)$, is obtained experimentally for the linear work hardening stage in aluminum (Fig. $3 \mathrm{c}$ ). It is described by the logistic function ${ }^{19}$ as

$$
\lambda(\delta)=\lambda_{0}+\frac{a_{1} / a_{2}}{1+C \exp \left(-a_{1} \delta\right)},
$$

where $\lambda_{0}, a_{1}$ and $a_{2}$ are empirical constants and $C$ is an integration constant. Function (3) is the solution of the differential equation $d \lambda=\left(a_{1} \lambda-a_{2} \lambda^{2}\right) \cdot d \delta^{19}$. Experimental data analysis suggests that the autowave length depends weakly on the grain size. Thus an increase in the value $\delta$ from $10^{-5}$ to $10^{-2} \mathrm{~m}$ would cause the value $\lambda$ to increase from $\sim 6 \cdot 10^{-3} \mathrm{~m}$ to $\sim 1.6 \cdot 10^{-2} \mathrm{~m}$.

\subsection{Introduction of elastic-plastic invariant}

At first glance it would seem that Eqs. (1), (2) and (3) are not interrelated; however, we are going to take a good look at these relationships to find out what relation binds them. With this aim in view, quantitative data processing of deformation patterns was performed for studied materials. It has been found that the auto wave characteristics, $\lambda$ and $V_{a w}$, taken together with the spacing, $X$, and the transverse elastic wave rate, $V_{t}$, make up the following ratio (see Table 1)

$$
\frac{\lambda \cdot V_{a w}}{\chi \cdot V_{t}} \equiv \frac{\lambda}{\chi} \cdot \frac{V_{a w}}{V_{t}}=\text { const }=\hat{Z}
$$

The calculated data suggest that the average quantity $\langle\hat{Z}\rangle=2 / 3 \pm 1 / 4$. Equation (4) is called 'elastic-plastic invariant' by virtue of the fact that it relates the characteristics of elastic waves, $X$ and $V$, to the characteristics of localized plastic flow autowaves, $\lambda$ and $V_{a w}$. The elastic waves are involved in the elastic stress redistribution by the deformation, while the localized plasticity autowaves are in point of fact deformation pattern evolution. Hence, Eq. (4) relates the above two types of processes. This reasoning may play a strategic role in envisaging new conceptual representations of the nature of localized plastic deformation. As is shown in Fig. 4, invariant (4) possesses universality: it is valid for the autowaves pro pa gating at the easy glide and linear stages of work hardening as well as for the motion of individual dislocations ${ }^{20}$.

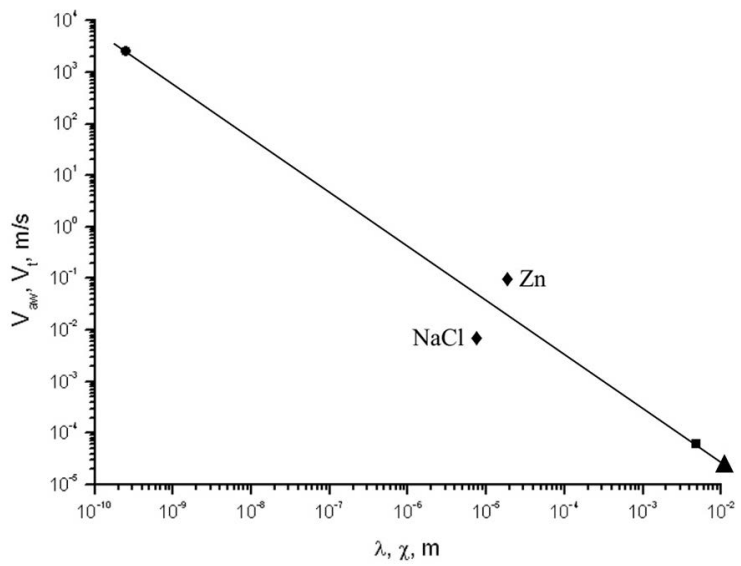

Figure 4. The meaning of the elastic-plastic invariant $•$ elastic deformation stage; $\boldsymbol{\|}$ linear work hardening tage; $\boldsymbol{\Delta}$ easy glide stage; $\bullet$ individual dislocation motion

In view of $V_{t}^{2} \approx X^{2} \omega_{D}^{2} \approx E / \rho$ (here $\omega_{D}$ is the De bye frequency; $\rho$, material density; $E \approx X^{-1} \cdot d^{2} W / d u^{2}$, elastic modulus; $u<<X$, displacement and $W(u)$, lattice po tential function ${ }^{21}$ ), invariant (4) is rearranged as

$$
V_{a w} \lambda \approx \hat{Z} \frac{V_{t}^{2}}{\omega_{D}} \approx \hat{Z} \cdot \frac{d^{2} W / d u^{2}}{\left(\omega_{D} \chi\right) \rho} \approx \hat{Z} \frac{d^{2} W / d u^{2}}{\xi_{1}},
$$

\begin{tabular}{|c|c|c|c|c|c|c|c|c|c|c|c|c|}
\hline \multirow{2}{*}{ Terms of invariant $\times 10^{7} \mathrm{~m}^{2} \cdot \mathrm{s}^{-1}$} & \multicolumn{12}{|c|}{ Metals } \\
\hline & $\mathrm{Cu}$ & $\mathrm{Zn}$ & $\mathrm{Al}$ & $\mathrm{Zr}$ & $\mathrm{Ti}$ & $\mathrm{V}$ & $\mathrm{Nb}$ & $\gamma-\mathrm{Fe}$ & $\alpha-\mathrm{Fe}$ & $\mathrm{Ni}$ & Co & $\mathrm{Sn}$ \\
\hline$\lambda \cdot V_{a w}$ & 3.6 & 3.7 & 7.6 & 2.9 & 3.5 & 2.8 & 1.8 & 2.1 & 2.3 & 2.1 & 3.0 & 2.8 \\
\hline$\chi \cdot V_{t}$ & 4.8 & 5.2 & 7.5 & 5.5 & 6.6 & 6.1 & 5.2 & 6.9 & 6.7 & 6.5 & 6.0 & 5.3 \\
\hline \multirow{2}{*}{ Terms of invariant $\times 10^{7} \mathrm{~m}^{2} \cdot \mathrm{s}^{-1}$} & \multicolumn{6}{|c|}{ Alkali halide crystals } & \multicolumn{6}{|c|}{ Rocks } \\
\hline & \multicolumn{2}{|c|}{$\mathrm{KCl}$} & \multicolumn{2}{|c|}{$\mathrm{NaCl}$} & \multicolumn{2}{|c|}{$\mathrm{LiF}$} & \multicolumn{3}{|c|}{ Marble } & \multicolumn{3}{|c|}{ Sandstone } \\
\hline$\lambda \cdot V_{a w}$ & \multicolumn{2}{|c|}{3.0} & \multicolumn{2}{|c|}{3.1} & \multicolumn{2}{|c|}{4.3} & \multicolumn{3}{|c|}{1.75} & \multicolumn{3}{|c|}{0.6} \\
\hline$\chi \cdot V_{t}$ & \multicolumn{2}{|c|}{7.0} & \multicolumn{2}{|c|}{7.5} & \multicolumn{2}{|c|}{8.8} & \multicolumn{3}{|c|}{3.7} & \multicolumn{3}{|c|}{1.5} \\
\hline
\end{tabular}

where the value $\zeta_{1}=\left(\omega_{D} X\right) \rho=\mathrm{V}_{\mathrm{t}} \rho$ is the medium's specific acoustic resistance ${ }^{22}$.

Table 1. Database for introducing elastic-plastic invariant (4). 


\subsection{Consequences of the elastic-plastic invariant}

Eqs. (1) - (3) can be derived from Eq. (4), which suggests that the elastic-plastic invariant plays an important role in the development of autowave concept of localized plasticity.

First, Eq. (4) is differentiated with respect to deformation, $\varepsilon$, as

$$
\lambda \frac{d V_{a w}}{d \varepsilon}+V_{a w} \frac{d \lambda}{d \varepsilon}=\hat{Z} \cdot \chi \frac{d V_{t}}{d \varepsilon}+\hat{Z} \cdot V_{t} \frac{d \chi}{d \varepsilon} .
$$

By writing the above expression with respect to $V_{a w}$, we obtain

$$
V_{a w}=\left(\frac{d \lambda}{d \varepsilon}\right)^{-1}\left(\hat{Z} \cdot \chi \frac{d V_{t}}{d \varepsilon}+\hat{Z} \cdot V_{t} \frac{d \chi}{d \varepsilon}-\lambda \frac{d V_{a w}}{d \varepsilon}\right)
$$

The value $X$ is unaffected by the deformation; hence, $\hat{Z} \cdot V_{t} \cdot d X / d \varepsilon=0$. Consequently,

$$
V_{a w}=\hat{Z} \chi \cdot \frac{d V_{t}}{d \lambda}-\lambda \cdot \frac{d V_{a w}}{d \lambda}
$$

A common transformation of Eq. (8) yields

$$
V_{a w}=\hat{Z} \chi \cdot \frac{d V_{t}}{d \lambda}-\chi \cdot \frac{d V_{a w}}{d \lambda} \cdot \frac{\lambda}{\chi} \approx V_{0}+\frac{\Xi}{\theta},
$$

which coincides with Eq. (1). The coincidence is due to the fact that $d V_{a w} / d \lambda<0$; besides, the work hardening coefficient, $\theta$, is given by the ratio of two structural parameters having the dimension of length, i.e. $\lambda$ and $X<<\lambda^{23-25}$; hence, $\theta \sim X / \lambda$.

Let Eq. (4) be written as

$$
V_{a w}=\frac{\Theta}{\lambda}=\frac{\Theta}{2 \pi} \cdot k,
$$

where $\Theta \hat{Z}=X V_{t}$. In view of $V_{a w}=d \lambda / d T=d \omega / d k$, we obtain $d \omega=(\Theta / 2 \pi) \cdot k \cdot d k$. Thus we obtain

$$
\int_{\omega_{0}}^{\omega} d \omega=\frac{\Theta}{2 \pi} \int_{0}^{k-k_{0}} k . d k .
$$

Hence, dispersion law of quadratic form is given as

$$
\omega=\omega_{0}+\frac{\Theta}{4 \pi}\left(k-k_{0}\right)^{2} \text {. }
$$

Thus, Eq. (12) is equivalent to Eq. (2), if $\Theta / 4 \pi \Xi \alpha$.

Now we write Eq. (4) of the form

$$
\lambda=\hat{Z} \chi \cdot \frac{V_{t}}{V_{a w}}
$$

By taking into account the dependence of rates $\mathrm{V}_{\mathrm{t}}$ and $\mathrm{V}_{\mathrm{aw}}$ on the grain size, $\delta^{5}$, differentiation of Eq. (13) is performed with respect to $\delta$ as

$$
\begin{gathered}
\frac{d \lambda}{d \delta}=\hat{Z} \chi \cdot \frac{d}{d \delta}\left(\frac{V_{t}}{V_{a w}}\right)= \\
\hat{Z} \chi \cdot\left(\frac{V_{a w} \cdot d V_{t} / d \delta-V_{t} \cdot d V_{a w} / d \delta}{V_{a w}^{2}}\right)
\end{gathered}
$$

Transformation of Eq. (14) yields the following differential equation

$$
d \lambda=\hat{Z} \chi \cdot\left(\frac{d V_{t}}{d \delta} \cdot \frac{1}{V_{a w}}-V_{t} \frac{d V_{a w}}{d \delta} \cdot \frac{1}{V_{a w}^{2}}\right) \cdot d \delta
$$

which can also be written as

$$
d \lambda=\left(a_{1} \lambda-a_{2} \lambda^{2}\right) \cdot d \delta
$$

where $a_{1}=\frac{1}{V_{t}} \cdot \frac{d V_{t}}{d \delta}=\frac{d \ln V_{t}}{d \delta}$ and $a_{2}=\frac{1}{\hat{Z} \chi V_{t}} \cdot \frac{d V_{a w}}{d \delta}$, since $V_{a w}=\hat{Z} \chi V_{t} \cdot \frac{1}{\lambda}$. Evidently, the solution of Eq. (16) coincides with Eq. (3).

Consider also the other consequences of the invariant. Let Eq. (4) be written as

$$
\chi / \lambda=\hat{Z}\left(V_{a w} / V_{t}\right)
$$

where the plastic deformation $\varepsilon \approx \lambda / X>>1$. By applying the operator $\partial / \partial t=D_{\varepsilon} \cdot \partial^{2} / \partial x^{2}$ to the right and left parts of Eq. (17), consequently, we obtain

$$
\frac{\partial \varepsilon}{\partial t}=-\hat{Z} D_{\varepsilon}\left(-V_{i} \cdot \frac{\partial^{2} V_{a w}^{-1}}{\partial \chi^{2}}+V_{a w}^{-1} \cdot \frac{\partial^{2} V_{t}}{\partial \chi^{2}}\right) .
$$

The ultrasound propagation rate depends weakly on the plastic deformation, $\varepsilon^{26}$; hence, $\mathrm{V}_{\mathrm{t}} \approx$ const. In this case, we obtain

$$
\frac{\partial \varepsilon}{\partial t}=-\hat{Z} D_{\varepsilon} V_{t} \frac{\partial^{2} V_{a w}^{-1}}{\partial \chi^{2}}+D_{\varepsilon} \frac{\partial^{2} \varepsilon}{\partial \chi^{2}},
$$

which is equivalent to the following differential equation for the deformation rate

$$
\dot{\varepsilon}=f(\varepsilon, \sigma)+D_{\varepsilon} \varepsilon^{\prime \prime},
$$

where the coefficient $D_{\varepsilon}$ has the dimension $\mathrm{L}^{2} \cdot \mathrm{T}^{-1}$. Equation (20) has the form of the reaction-dif fu si on equation for a concentration, $\zeta=\varphi(\zeta)+D \zeta^{\prime \prime}$, which is obtained when the nonlinear function, $\varphi(\zeta)$, is entered into the right part of Fick's second law for diffusion $\dot{\zeta}=D \zeta^{\prime \prime}{ }^{27}$. Equation (20) falls into the category of nonlinear relations employed for describing autowave processes, which occur in different kinds of open systems, provided that adequate variables are chosen for solving the problem ${ }^{28,29}$. In what follows, Eq. (20) is discussed in detail.

\subsection{Plastic deformation viewed as autowave generation process}

By elaborating the autowave concept, we must consider the dependence of autowave patterns on the work hardening law acting at a given plastic flow stage. To make out individual flow stages on the curve $\sigma(\varepsilon)$, the Lüdwick equation ${ }^{30}$ is used

$$
\sigma(\varepsilon)=\sigma_{0}+K \varepsilon^{\prime \prime}
$$


where $K$ is a hardening modulus; $\sigma_{0}=$ const and $0 \leq n \leq 1$ is a hardening exponent which varies discretely. To verify whether Eq. (21) is operative, the Kocks-Mecking method ${ }^{31}$ was used for recognizing plastic flow stage. The results obtained in both cases were found to fit neatly. The in dividual work hardening stages can be distinguished on the flow curve subject to the condition that $n=(1 n \varepsilon)^{-1} \cdot\left[1 n\left(\sigma-\sigma_{0}\right)-1 n K\right] \approx$ const. A total of four flow stages have been distinguished, i.e. easy glide/yield plateau, linear work hardening stage, parabolic work hardening stage and pre-failure stage. Likewise, the possible macro-locali zation patterns are limited in number: a total of four types of localized plasticity patterns are found to emerge in the deforming sample. Table 2 presents the localization patterns matched against the autowave modes. Evidently, a one-to-one correspondence exists between the autowave modes and the respective plastic flow stages (Table 2 and Fig. 5). In view of the above, the sample tested in constant-rate tension can be regarded as a universal generator of autowaves ${ }^{32}$ that requires no maintenance of temperature or reagent concentration as is the case with, e.g. chemical reactors ${ }^{6,19}$.

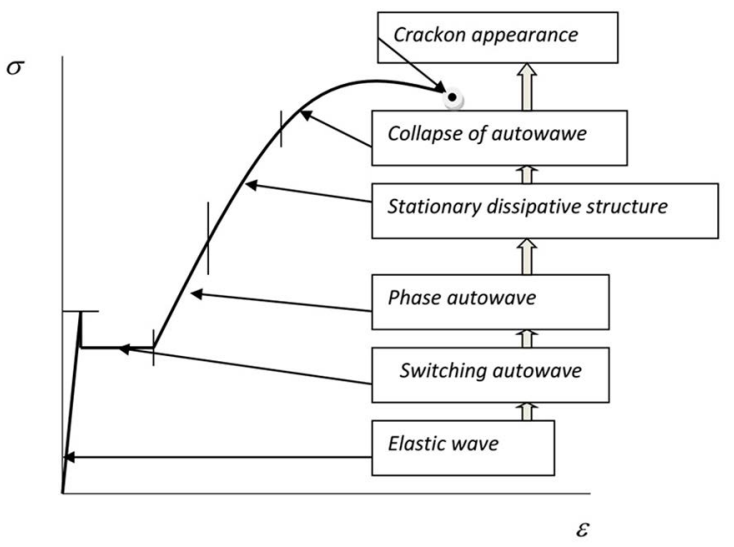

Figure 5. Wave and autowave processes involved in deformation

We conclude this Section by saying that the above analysis provides a unified explanation for the autowave nature of plasticity; hence, substantial revision of traditional notions in this field is required. Now that the work hardening process is regarded as evolution of the autowave modes, deformation kinetics analysis must involve different principles so as to formulate a new viewpoint of the nature of multi-stage plastic flow.
The autowave plastic deformation exhibits regular features, which are consequences of Eq. (4); all the empirical coefficients in Eqs. (1) - (3) have been assigned a physical meaning. In the frame of this concept the micro-scale level is related to the macro-scale features of localized plastic deformation by Eq. (4), which should be regarded as 'master equation' of the plasticity theory being developed.

\section{The two-component model of plastic flow}

\subsection{Nonlinear equations for localized plastic flow}

In general, the processes involved in self-organization are conventionally regarded as an interplay of an activator and a damper ${ }^{6,28}$. An understanding of how the both factors relate to the localized plastic flow could illuminate the roles they play. It is therefore assumed that the plastic deformation, $\varepsilon$, is an activator and the elastic stresses, $\sigma$, a damper.

It would be reasonable to describe the kinetics of the activator using Eq. (20), which was inferred as a consequence of invariant (4). In turn, the stress relaxation kinetics can be described using the Euler equation for viscous liquid flux ${ }^{33}$, which has the form

$$
\frac{\partial}{\partial t} p v_{i}=-\frac{\partial}{\partial x_{k}} \prod_{i k}
$$

where $\prod_{i k}=p \delta_{i k}+p v_{i} v_{k}-\sigma_{v i s}=\sigma_{i k}-p v_{i} v_{k} \quad$ is momentum flux density tensor; $\delta_{\mathrm{ik}}$, unit tensor; $p$, pressure; $v_{i}$ and $v_{k}$, flux velocity components. The stress tensor $\sigma_{i k}=-$ $p \delta_{i k}+\sigma_{v i s}$ is the sum of elastic and viscous stresses, respectively, i.e. $\sigma_{e l}=-p \delta_{i k}$ and $\sigma_{\text {vis }}$. In the case of plastically deforming medium, $\sigma=\sigma_{e l}+\sigma_{v i s}$, i.e. $\dot{\sigma}=\dot{\sigma}_{e l}+\dot{\sigma}_{v i s}$. It is reported in ${ }^{31,34}$ that $\dot{\sigma}_{e l} \equiv g(\sigma, \varepsilon)=-M p_{m} b^{2}(\sigma / B)=-M p_{m} b V_{d i s l} \sim V_{d i s l}$ (here $\mathrm{M}$ is elastic modulus for the sys tem 'sample - testing machine'; $\rho_{m}$ is mobile dislocation density and $V_{\text {disl }}$ is dislocation motion rate).

Due to the inhomogeneous inter nal elastic strain field, viscous stresses, $\sigma_{\text {vis }}$, will form in the deforming medium. The viscous stresses are re la ted to the variations in the elastic wave rate which are linear with respect to stresses, i.e. $V_{t}=V_{t 0}+\kappa \sigma^{26}$. Here $V_{t 0}$ is the transverse elastic wave rate in the absence of stres ses and $\kappa=$ const. Assuming that $\sigma_{v i s}=B \partial V / \partial x$ (here $B$ is medium's dynamic viscosity), we can write $\partial \sigma_{v i s} / \partial t=V_{t} \partial / \partial x(B \partial V / \partial x)=B V_{t} \partial^{2} V / \partial x^{2}$.

Table 2. Plastic flow stages matched against autowave modes.

\begin{tabular}{lcc}
\hline \multicolumn{1}{c}{ Work hardening stage } & The exponent $n$ in Eq. (21) & Autowave mode \\
\hline Yield plateau or easy glide stage & $\sim 0$ & Switching autowave \\
\hline Linear work hardening stage & 1 & Phase autowave \\
\hline Parabolic work hardening stage & $1 / 2$ & Stationary dissipative structure \\
\hline Pre-failure stage & $<1 / 2$ & Collapse of autowave \\
\hline
\end{tabular}


Hence, the relaxation rate is given for viscous stres ses as $\partial \sigma_{v i s} / \partial t=B V_{t} \partial^{2} V / \partial x^{2}=B \kappa V_{t} \partial^{2} \sigma / \partial x^{2}$. Apparently,

$$
\partial \sigma / \partial t=g(\varepsilon, \sigma)+D_{\sigma} \partial^{2} \sigma / \partial x^{2}
$$

where $D_{\sigma}=B \kappa V_{t}$ is a transport coefficient having the dimension $\mathrm{L}^{2} \cdot \mathrm{T}^{-1}$. Thus, Eq. (23) can be used to describe the damper kinetics in the deforming medium by virtue of the fact that $D_{\sigma} \equiv D_{\text {damp }}$. The right part of Eq. (23) is the sum of relaxation rates of elastic and viscous stresses, $\dot{\sigma}_{e}=g(\varepsilon, \sigma)$ and $\dot{\sigma}_{\text {vis }}=\mathrm{D}_{\sigma} \cdot \partial^{2} \sigma / \partial \mathrm{x}^{2}$, respectively. The nonlinear function $g(\varepsilon, \sigma)$ accounts for the elastic stress redistribution among neighboring material micro-volumes ahead of the moving plastic deformation front and the diffusion-like term $D_{\sigma} \cdot \partial^{2} \sigma / \partial x^{2}$ is responsible for elastic stress redistribution among material volumes via macro-scale stochastic processes.

The above line of mathematical reasoning can be made more accessible by suggesting that the flow rates $\dot{\varepsilon}$ and $\dot{\sigma}$ incorporate both the 'hydrodynamic' and 'diffusion' com ponents. The hydrodynamic components are given by the nonlinear functions $f(\varepsilon, \sigma) \sim V_{\text {disl }}$ and $g(\sigma, \varepsilon) \sim V_{\text {disl }}$ from Eqs. (20) and (23), correspondingly. These have to do with the steady moti on of deformation fronts along the sample, with the local stress concentrators occurring on the fronts being activated one by one. The diffusion components are given by the terms $D_{\varepsilon} \partial^{2} \varepsilon / \partial x^{2}$ and $D_{\sigma} \partial^{2} \sigma / \partial x^{2}$ from Eqs. (20) and (23). These are responsible for the deformation initiated in material volumes at macroscopic distance $\sim \lambda$ from the active deformation front.

Thus, the resultant system of equations

$$
\left\{\begin{array}{l}
\dot{\varepsilon}=f(\varepsilon, \sigma)+D_{\varepsilon} \varepsilon^{\prime \prime} \\
\dot{\sigma}=g(\varepsilon, \sigma)+D_{\sigma} \sigma^{\prime \prime}
\end{array}\right.
$$

may be used for autowave mode analysis, provided that the nonlinear functions $f(\varepsilon, \sigma)$ and $g(\sigma, \varepsilon)$ in explicit form are available. Equations (20), (23), a system of Eq. (24a, b) and the reasoning involved in the derivation thereof served the basis for the development of two component model of plasticity. Two versions of the same model are discussed below.

\subsection{The two-component model. The autowave version}

Note that the coefficients $D_{\varepsilon}$ and $D_{\sigma}$ from the system of Eqs. (24a) and (24b) have dimensions $\mathrm{L}^{2} \cdot \mathrm{T}^{-1}$, which coincide with those of the products $\lambda \cdot V_{a w}$ and $X \cdot V_{t}$ from invariant (4). Hence,

$$
\lambda \cdot V_{a w} \equiv D_{\varepsilon}
$$

and

$$
\chi \cdot V_{t} \equiv D_{\sigma}
$$

The above suggests that invariant (4) is equivalent to the ratio $D_{\varepsilon} / D_{\sigma}=2 / 3<1$, i.e. $D_{\sigma} \equiv D_{\text {damp }}>D_{\varepsilon} \equiv D_{\text {activ }}$. The condition $\mathrm{D}_{\text {damp }}>\mathrm{D}_{\text {activ }}$ is a prerequisite for auto wave ge ne ration in the active medium ${ }^{6}$. The autowave structure formation should be regarded as a basic attribute of the self-organizing active medium ${ }^{6}$. In a general case, the likelihood that self-organization processes will be initiated in the active medium depends on whether the medium itself is capable of separating spontaneously into information and dynamic subsystems ${ }^{35}$. The main features of plastic flow might be explained by assuming that the information subsystem is related to acoustic emission pulses generated by dislocation shears and the dynamic subsystem, to shears proper ${ }^{36}$. The evolution of two subsystems is described by Eqs. ${ }^{(20)}$ and ${ }^{(23)}$.

Based on the assumptions above, a two-component model of plastic flow is proposed which operates in accordance with the scheme presented in Fig. 6. Due to the stress concentrator decay (1), stress relaxation will occur which causes generation of acoustic emission pulses. The stress relaxation results in the liberation of energy which is absorbed by the remaining stress concentrators which act as energy-sink (2). This phenomenon is known as acoustic-plas tic effect ${ }^{37}$. As new stress relaxation acts are initiated, shear processes will continue to occur in the dynamic subsystem, generating thereby a series of acoustic pulses. Thus the basic idea of the model being developed is that the acoustic emission and the acousticplastic effect are in no way interdependent. This issue has been neglected thus far. In support of this interpretation one can argue that the elastic-plastic invariant is given by Eq. (5), which contains the term $\xi_{1}=V_{t} \rho$ for medium's acoustic resistance.

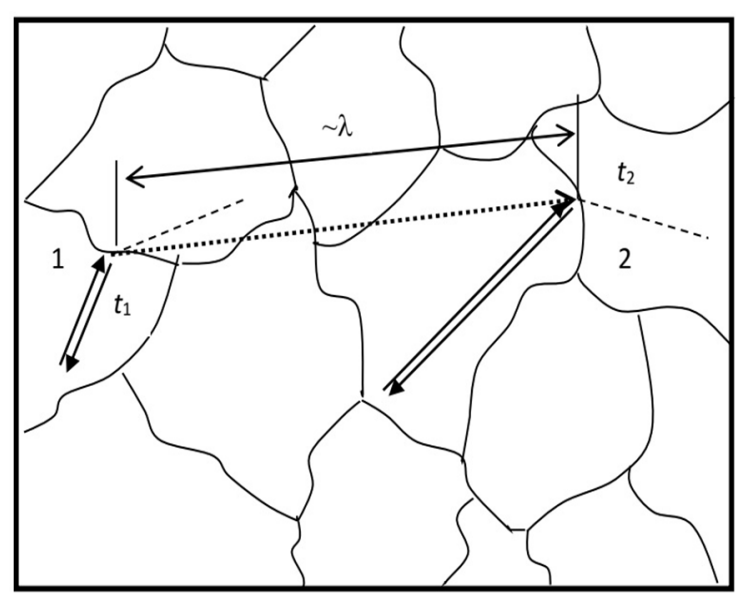

Figure 6. A schematic of the generation of localized plasticity fronts $\rightleftarrows$ dislocation shears (stress concentrators); - - - - - shears due to decay of stress concentrators; ............... elastic pulse trajectory 
To verify the model's validity, the expectation times, $\tau^{*}$ and $\tau^{* *}$, are estimated for the thermally activated relaxation acts $^{38}$. In the event that the external stress alone is operating,

$$
t^{*}=\omega_{D}^{-1} \exp \left(\frac{U_{0}-\gamma \sigma}{k_{B} T}\right)
$$

in the event that both the external stress and the acoustic pulse are operating,

$$
\begin{gathered}
\tau^{* *} \approx \omega_{D}^{-1} \exp \left[\frac{U_{0}-\gamma \sigma-\Delta U_{a c}}{k_{B} T}\right]= \\
\omega_{D}^{-1} \exp \left[\frac{U_{0}-\gamma\left(\sigma+\varepsilon_{a c} E\right)}{k_{B} T}\right] .
\end{gathered}
$$

In Eqs. (26) and (27) the activation enthalpy, $U_{0}-\gamma \sigma \approx$ $0.5 \mathrm{eV}^{38}$. Due the action of acoustic pulse having amplitude, $\varepsilon_{a c} \approx 2 \cdot 10^{-6}$, reduction in the activation enthalpy is $\Delta U_{a c} \approx \gamma \varepsilon_{a c} E$ $\approx 0.1 \mathrm{eV}$ and $k_{B} T \approx 1 / 40 \mathrm{eV}$. Under the above conditions, $\tau^{*}$ $\approx 5 \cdot 10^{-5} \mathrm{~s}$ and $\tau^{* *} \approx 9 \cdot 10^{-7} \mathrm{~s}<<\tau^{*}$. The estima te, rough as it is, evidently speaks for the proposed model. It is thus proved that the events occurring in the acoustic (information) and dislocation (dynamic) subsystems are interrelated.

The effect of transverse ultrasound wave splitting in the field of elastic stresses can be used to estimate the autowave length ${ }^{39}$. Assume that a transverse ultrasound pulse is emitted by an elementary shear. The maximal power in the acoustic emission spectrum corresponds to the frequency, $\omega_{a} \approx 10^{6} \mathrm{~Hz}^{37}$. Due to the pulse splitting occurring in an elastically stressed area, two orthogonal polarized waves will form; these have lengths $\varsigma_{1}=v_{1} / \omega_{a}$ and $\varsigma_{2}=v_{2} / \omega_{\mathrm{a}}$ and propagation rates $v_{1} \neq v_{2}$. A difference in the wavelengths is given in ${ }^{39}$ as

$$
\delta S=S_{2}-S_{1}=\frac{v_{2}-v_{1}}{\omega_{a}}=\frac{\sigma_{2}-\sigma_{1}}{2 \omega_{a} p V_{t}}
$$

An estimate of the same values can be obtained by assuming that a difference in principal normal stresses, $\sigma_{2}-\sigma_{1} \approx 10^{8} \mathrm{~Pa}$; material density, $\rho \approx 5 \cdot 10^{3} \mathrm{~kg} / \mathrm{m}^{3}$ and sound rate, $V \approx 3 \cdot 10^{3} \mathrm{~m} / \mathrm{s}$. Using this line of reasoning, we obtain that $\delta \varsigma \approx 10^{-4} \mathrm{~m}$. This rough estimate suggests that the maximal energy of the elastic wave is accumulated at distance $\sim \varsigma^{2} / \delta \varsigma \approx 10^{-2} \mathrm{~m} \sim \lambda$ from the pul se origin. This is a plausible explanation for the fact that the new localized plasticity front emerges at distance $\sim \lambda$ from the existing deformation front.

\subsection{The two-component model. The quasi-particle version}

The above numerical analysis demonstrates that the products $\lambda \cdot V_{a w} \cdot \rho \cdot X^{3}$ obtained for fourteen metals are close to the quantum Planck constant $h=6.626 \cdot 10^{-34} \mathrm{~J} \cdot \mathrm{s}{ }^{40}$. On the strength of da ta presented in Table 3 , we write

$$
\lambda_{1} \cdot V_{a w, i} \cdot \rho i \cdot \chi_{i}^{3}=h_{i}
$$

where $1 \leq \mathrm{i} \leq 14$. The average value $\langle h\rangle(6.95 \pm 0.48) \cdot 10^{-34} \mathrm{~J} \cdot \mathrm{s}$; hence, the ratio $\langle h\rangle / \mathrm{h}=1.05 \pm 0.07 \approx 1$. Without doubt, this intriguing result requires statistical verification. To decide whether the values $\langle h\rangle$ and $h$ are equal or different, they were compared with the help of Student's $\hat{t}$-criterion ${ }^{41}$, which was calculated from the formula

$$
\hat{t}=\frac{\langle h\rangle-h}{\sqrt{\hat{\sigma}^{2}}} \cdot \sqrt{\frac{n_{1} \cdot n_{2}}{n_{1}+n_{2}}},
$$

where $n_{1} 14$ is the number of estimates of $h_{i}$ from Eq. (32) and $n_{2}=1$. This suggests that high-accuracy measurements were performed in the absence of dispersion. The estimate of ove rall dispersion, $\hat{\sigma}^{2}$, can be made for the quantities $\langle h\rangle$ and $h^{41}$ as

$$
\hat{\sigma}^{2}=\frac{\sum_{i=1}^{n_{1}}(h i-\langle h\rangle)^{2}+\sum_{1}^{1}(h-h)^{2}}{n_{1}+n_{2}-2}=
$$

The calculation of the $\hat{t}$-criterion demonstrates that the values $\langle h\rangle$ and $h$ really belong to the sampling from one and the same general population with the probability higher than 95\%. Thus, Eq. (29) actually determines the Planck constant. This leads us to believe that quantum mechanics prin ciples are also suitable for investigations performed in the frame of plastic deformation physics.

This finding led us to believe that a hypothetical a quasiparticle might be introduced for addressing the localized plasticity autowave. This procedure is conventionally applied in solids physics ${ }^{42}$. By omitting the index ' $i$ ' from Eq. (29), we obtain

$$
\rho \cdot \chi^{3}=\rho \Omega=m_{e f}=h / \lambda V_{a w}
$$

which is easily identified with the well-known de Broglie

\begin{tabular}{|c|c|c|c|c|c|c|c|c|c|c|c|c|c|c|}
\hline Metals & $\mathrm{Mg}$ & Al & $\mathrm{Ti}$ & $\mathrm{Zr}$ & $\mathrm{Nb}$ & $\mathrm{Cu}$ & $\mathrm{Zn}$ & In & Sn & V & $\gamma-\mathrm{Fe}$ & $\alpha-\mathrm{Fe}$ & $\mathrm{Ni}$ & Co \\
\hline$h \cdot 10^{34}, \mathrm{~J} \cdot \mathrm{s}$ & 4.9 & 6.2 & 6.9 & 6.1 & 5.1 & 11.9 & 9.3 & 10.1 & 7.5 & 3.5 & 6.3 & 6.3 & 6.1 & 7.1 \\
\hline $\begin{array}{c}\mathrm{m}_{\mathrm{a}-\mathrm{l}} \\
\text { a.m.u. }\end{array}$ & 4.0 & 0.5 & 1.1 & 2.0 & 2.3 & 1.8 & 1.1 & 1.5 & 1.3 & 1.4 & 1.8 & 1.8 & 1.9 & 1.3 \\
\hline
\end{tabular}
equation, which gives the effective mass of a quasiparticle moving with velocity $V_{a w}$ as $m_{e f} \approx \rho X^{343}$. Thus, a quasi-particle is postulated which corresponds to the localized plasticity autowave; its effective mass is given as $m_{a-l}=h / \lambda V_{a w}$. The hypothetical quazi-particle has been called

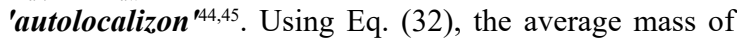
the autolocalizon was calculated for fourteen me tals; the

Table 3. Planck constant values, $h$, and autolocalizon masses, $\mathrm{ma}_{-1}$, calculated for studied metals. 
value obtained, $\left\langle m_{a-l}\right\rangle=1.7 \pm 0.2$ a.m.u. An attempt of similar kind is worthy of notice. Thus the authors in ${ }^{46}$ introduced a quasi-particle, so-called 'crackon', which was identified with the tip of growing brittle crack. It was also attempted to introduce so-called 'frustron', which might be appropriate for descriptions of the initial stage of lattice defect generation ${ }^{47}$.

A striking analogy can be drawn between the auto localizons and the 'rotons'. The latter quasi-particles were introduced in the theory of liquid $\mathrm{He}^{4}$ super fluidity ${ }^{48}$. The auto localizon and the ro ton have a dispersion law of quadratic form; the effective mass of roton, $m_{\text {rot }} \approx 0.64$ a.m.u. ${ }^{48}$, is close to that of auto localizon. In view of the above, the given approach could be elaborated in more detail.

Let Eq. (29) be written as

$$
\lambda V_{a w} \rho=h / \chi^{3}
$$

where both terms have the dimension of dynamic viscosity, i.e. $\mathrm{M} \cdot \mathrm{L}^{-1} \cdot \mathrm{T}^{-1} \equiv \mathrm{Pa} \cdot \mathrm{s}$. Using Eq. (33), calculations were performed for all studied metals; the resultant value, $\lambda \mathrm{V}_{\mathrm{aw}} \rho=\mathrm{h} / \mathrm{X}^{3} \approx 5 \cdot 10^{-4} \mathrm{~Pa} \cdot \mathrm{s}$. The latter value is close to the coefficient of dislocation drag, $B$, obtained for quasi-viscous dislocation motion rate $\mathrm{V}_{\text {disl }} \approx(\mathrm{b} \sigma) / \mathrm{B}^{34}$. It is thus concluded that elastic-plastic invariant (4) might be employed in the frame of alternative approach. In view of the equality

$$
\lambda V_{a w}=h /\left(p \cdot \chi^{3}\right)=h / m_{a-1},
$$

we may write, analogously,

$$
\chi V_{t}=h / m_{p h}
$$

where $m_{p h}$ is taken to be phonon mass. Now invariant (4) may be written as

$$
\hat{Z} \cdot \frac{h}{\lambda V_{a w}}=\frac{h}{\chi V_{t}}
$$

which reduces to the balance of masses as $2 m_{a-l}=3 m_{p h}$, i.e. three phonons would produce a pair of autolocalizons. This suggestion undoubtedly requires verification. It might be well to point out that si milar complex processes occurring in the phonon gas were described earlier ${ }^{49,50}$.

In the frame of quasi-particle approach the deforming medium might be viewed as a mixture of phonons and autolocalizons. It is thus suggested that the random walk of the Brownian particle is ${ }^{48}$

$$
S \approx \sqrt{\frac{K_{B} T}{\pi B r_{a-1}}} t
$$

where the time, $\tau=2 \pi / \omega \approx 10^{3} \mathrm{~s}$; the dynamic viscosity of the phonon gas, $B \approx 5 \cdot 10^{-4} \mathrm{~Pa} \cdot \mathrm{s}$ (see above) and $k_{B} T 1 / 40 \mathrm{eV}$ for $T=300 \mathrm{~K}$. Hence, the effective size of autolocalizon, $r_{a-l} \approx \sqrt[3]{\Omega} \approx \chi \approx 10^{-10} \mathrm{~m}$ and the quasi-particle walk, $\mathrm{S} \approx 10^{-2} \mathrm{~m} \approx \lambda$, which agrees with both the experimental value and that calculated from Eq. (28). By rewriting Eq. (37) as

$$
\frac{S^{2}}{t} \approx \frac{k_{B} T}{\pi B r_{a-1}}
$$

we obtain $S^{2} / \tau \approx D_{\varepsilon} \approx 1.3 \cdot 10^{-7} \mathrm{~m}^{2} / \mathrm{s}$. The above suggests that the processes involved in the plastic flow can be addressed effectively in the frame of quasi-particle and auto wave approaches.

\section{Localized plasticity autowaves and dislocation theory}

\subsection{The autowave deformation and the Taylor- Orowan equation}

The problem this approach seeks to resolve is this: little is known about its relation to the dislocation theory which serves as the basis for the overwhelming majority of traditional models in plasticity physics ${ }^{23-25,51,52}$. Therefore, it is absolutely necessary to relate of the autowave equations derived herein to the dislocation mechanisms of plasticity. Note that the idea about quantization of dislocation deformation is in no way objectionable, since the Burgers vector, $\mathbf{b}=\mathbf{a}_{\mathbf{1}}+\mathbf{a}_{\mathbf{2}}+\mathbf{a}_{3}$, is usually considered as 'a quantum of shear deformation' and its components, $\boldsymbol{a}_{i}$, are topological quantum numbers ${ }^{50,53}$. Now consider the function $f(\varepsilon, \sigma)=\varepsilon^{\prime} \cdot D_{\varepsilon}^{\prime}$ in Eq. (20). For a homogeneous distribution of dislocations with average spacing $d, \varepsilon^{\prime} \approx d^{-1} \cdot(b / d) \approx b \cdot d^{-2} \approx b \rho_{m}$ (here $b / d$ is shear per dislocation and $d^{-2} \approx \rho_{m}$ is mobile dislocation density). Given $\mathrm{D}_{\varepsilon} \approx \mathrm{L} \cdot \mathrm{V}_{\text {disl }}$ (here $L \approx \alpha x$ is dislocation path and $V_{\text {disl }}=$ const, dislocation rate), we obtain $D_{\varepsilon}^{\prime}=\alpha V_{d i s l}$. Hence, Eq. (20) reduces to the equality

$$
\dot{\varepsilon}=\alpha b \rho_{m} V_{d i s l}+D_{\varepsilon} \varepsilon^{\prime \prime},
$$

where the first term from the right side coincides with the Taylor-Orowan equation for dislocation deformation, i.e. $\dot{\varepsilon}=b \rho_{m} V_{\text {disl }}$, which might be considered now as a special case of Eq. (39). However, Eq. (39) is applicable to more general cases, e.g. media having high dislocation density, provided the diffusion-like term $\mathrm{D}_{\varepsilon} \varepsilon^{\prime \prime}$ is added to the right side of the Taylor-Orowan equation. To clarify this purely formal procedure, we shall give some explanation of the condition to be met. Given $\dot{\varepsilon}=$ const by constant-rate tensile loading, the required level of dislocation flux, $\rho_{m} V_{d i s}$, has to be maintained. If the condition is not met, owing to, e.g. a decrease in mobile dislocation density, the medium will initiate deformation processes in front of the plasticity nucleus remote from the nuclei already in existence - otherwise sample fracture will occur. This representation provides a new way of tackling nonlinear problems connected with the nature and evolution of dislocation substructures ${ }^{24,25,52}$.

\subsection{Localized plasticity autowaves and work}




\section{hardening coefficient}

A number of well-established models link work hardening processes to the long- or short-range interaction of dislocations ${ }^{51,52}$. In what follows, the autowave mechanism has to be related to the work hardening phenomena in terms of plasticity physics. Assume that the work hardening coefficient is given as $\theta \approx W / Q^{23}$ (here $W \approx E b^{2} \rho s$ is the energy stored by plastic defor ma tion; $\rho_{\mathrm{s}}$, immobile dislocation density; $Q \approx \sigma b L \rho_{m}$, energy dissipated by mobile dislocati ons having density, $\rho m$, and path, $\mathrm{L}_{\mathrm{d}}$ ). Now it can be written

$$
\theta \approx \frac{E b^{2} \rho_{s}}{\sigma b L_{d} \rho_{m}} \approx \frac{b}{\varepsilon_{e} L_{d}} \cdot \frac{\rho_{s}}{\rho m},
$$

where $\varepsilon_{\mathrm{e}}=\sigma / \mathrm{E} ; \mathrm{L}_{\mathrm{d}}=\Lambda \cdot\left(\varepsilon-\varepsilon^{*}\right)^{-1} ; \Lambda$ depends on the kind of material ${ }^{24}$ and $\varepsilon^{*}$ is the strain for the onset of linear work hardening stage. It follows from Eqs. (1) and (40) that

$$
V_{a w} \approx \Xi \cdot \frac{\Lambda}{b} \cdot \frac{\varepsilon-\varepsilon^{*}}{\varepsilon_{e}} \cdot \frac{\rho_{m}}{\rho_{s}} .
$$

With growing density of immobile defects, $\rho_{s}$, the value $V_{a w}$ will grow less. With increasing amo unt of energy dissipated to he at, the deforming medium would warm up, which greatly increases the likelihood of thermally activated plastic deformation and of autowave rate growth.

Now let $d V_{a w} \sim L_{d}$; hence, we obtain ${ }^{24,25}$

$$
\theta \approx \sqrt{z b / 3 \wedge}
$$

where $z$ is the number of dislocations in a planar pileup. It follows from the above that $d V_{a w} \sim L_{d} \sim \Lambda \sim \theta^{-2}$. The data obtained for a range of materials suggest that $\varepsilon-\varepsilon * \approx$ const $t^{24}$; however, the value $\theta$ is found to vary for different materials, i.e. $d \theta \neq 0$. Then we can write

$$
d V_{a w} \approx z b\left(\varepsilon-\varepsilon^{*}\right)^{-1} \theta^{-2} d \theta .
$$

Integration of Eq. (43) yields $V_{a w} \sim \theta^{-1}$. Finally, a special case of Eq. (1) is written as

$$
V_{a w}=V_{0}^{*}+J / \theta^{*}
$$

where $\theta^{*}=d \sigma / d \varepsilon$ is a dimension characteristic of the plastic flow process (see above). The values calculated for alloyed single $\gamma$-Fe crystals are as follows: $V_{0}^{*} \approx 2.1 \cdot 10^{-5} \mathrm{~m} / \mathrm{s}$ and $J \approx 3.4 \cdot 10^{4} \mathrm{~Pa} \cdot \mathrm{m} / \mathrm{s}$. Let $J \approx \sigma V_{m}$ (here $\sigma$ is flow stress and $V_{m}$ is mobile grip rate). Since Pa $\mathrm{m} / \mathrm{s}=\mathrm{W} \cdot \mathrm{m}^{-2}$, the quantity $J$ acquires the mea ning of flux power from the loading machine through the sample. Given $\sigma \leq 10^{3} \mathrm{MPa}$ and $\mathrm{V}_{\mathrm{m}}=3.3 \cdot 10^{-6} \mathrm{~m} / \mathrm{s}$, we obtain $\mathrm{J} \leq 3.3 \cdot 10^{4} \mathrm{~W} \cdot \mathrm{m}^{-2} 54-56$.

Thus the work hardening process is addressed above in the frame of conventional dislocation model by assuming that a changeover in the work hardening stages is due to variation in the distribution of stress concentrators ${ }^{51}$. Hence, the same factor might be responsible for the generation of new autowave modes in the active medium.

\section{Conclusions}

Solids plasticity is addressed above in the frame of autowave concept. The given approach might fall far short of a completely theoretical prediction. In point of fact, it might be regarded as an attempt at envision of the complexities of the plastic flow process, which would enable formulation of contemporary views of this phenomenon. Hopefully, more rigorous techniques would be developed for tackling the problem of plasticity. Thus far we have the pleasure of bringing to your notice the following conclusions.

1. The localization behavior of plastic deformation has been studied for all the plastic flow stages. Localized plasticity patterns are found to emerge in the deforming medium. A total of four pat tern kinds have been recognized in studied materials. It is found that the kind of pattern observed for the given flow stage strictly corresponds to the work hardening mechanism involved in the deformation.

2. The kinetics of plastic deformation and stresses is described using autowave equations derived on the base of continuum media mechanics.

3. On the base of experimental data elastic-plastic deformation invariant is obtained, which is a master equation for plasticity mechanics. In the frame of autowave model the invariant serves to relate the characteristics of localized plasticity autowaves to those of elastic waves. The distinctive features of localized plasticity autowaves are described by the consequences from the invariant.

4. A two-component model of localized plasticity is proposed which is based on the assumption that shear processes interact with acoustic pulses. The autowave model is complemented by the quasi-particle model; the both versions are of equivalent status.

5. A relationships are established between the autowave representations and certain conventional dislocation models, which precludes any controversy between the proposed auto wave approach and the existing work hardening theories.

\section{Acknowledgements}

The work was performed within the Program of Fundamental Research of State Academies of Sciences for the period of 2013-2020 and was supported by Tomsk State University in the framework of the Competitiveness Improvement Program. 


\section{References}

1. Zuev LB. Wave phenomena in low-rate plastic flow of solids. Annalen der Physik. 2001;10(11-12):965-984.

2. Zuev LB. On the waves of plastic flow localization in pure metals and alloys. Annalen der Physik. 2007;16(4):286-310.

3. Zuev LB. Autowave mechanics of plastic flow in solids. Physics of Wave Phenomena. 2012;20(3):166-173.

4. Zuev LB, Barannikova SA. Plastic Flow Localization Viewed as an Auto-Wave Process Generated in Deforming Metals. Solid State Phenomena. 2011;172-174:1279-1283.

5. Seeger A, Frank W. Structure Formation by Dissipative Processes in Crystals with High Defect Densities. In: Kubin LP, Martin G, eds. Non-Linear Phenomena in Materials Science. Zurich: Trans Tech Publications; 1987. p. 125-138.

6. Haken H. Information and Self-Organization. A Macroscopic Approach to Complex Systems. 3rd ed. Berlin Heidelberg: Springer-Verlag; 2006.

7. Zuev LB. Autowave processes of the localization of plastic flow in active media subjected to deformation. Physics of Metals and Metallography. 2017;118(8):810-819.

8. Zuev LB. Entropy of localized plastic strain waves. Technical Physics Letters. 2005;31(2):89-90.

9. Hähner P. Theory of solitary plastic waves. Part I: Lüders bands in polycrystals. Applied Physics A. 1994;58(1):41-58.

10. Ananthakrishna G, Valsakumar MC. Repeated yield drop phenomenon: a temporal dissipative structure. Journal of Physics D: Applied Physics. 1982;15(12):L171.

11. Glazoff MV, Barlat F, Weiland H. Continuum physics of phase and defect microstructures: bridging the gap between physical metallurgy and plasticity of aluminum alloys. International Journal of Plasticity. 2004;20(3):363-402.

12. Aifantis EC. Gradient plasticity. In: Lemaitre J, ed. Handbook of Materials Behavior Models. New York: Academic Press; 2001. p. 281-307.

13. Aifantis EC. On the gradient approach - Relation to Eringen's nonlocal theory. International Journal of Engineering Science. 2011;49(12):1367-1377.

14. Aifantis EC. Gradient material mechanics: Perspectives and Prospects. Acta Mechanica. 2014;225(4-5):999-1012.

15. Acharia A, Beaudoin A, Miller R. New Perspectives in Plasticity Theory. Dislocation Nucleation, Waves, and Partial Continuity of Plastic Strain Rate. Mathematics and Mechanics of Solids. 2008;13(3-4):292-315.

16. Fressengeas C, Beaudoin AJ, Entemeyer D, Lebedkina T, Lebyodkin M, Taupin V. Dislocation transport and intermittency in the plasticity of crystalline solids. Physical Review B. 2009;79(1):014108.

17. Lebyodkin MA, Kobelev NP, Bougherira Y, Entemeyer D, Fressengeas C, Gornakov VS, et al. On the similarity of plastic flow processes during smooth and jerky flow: Statistical analysis. Acta Materialia. 2012;60(9):3729-3740.

18. Dodd RK, Eilbeck JC, Gibbon JD, Morris HC. Solitons and Nonlinear Wave Equations. London: Academic Press; 1982.
19. Scott A. Nonlinear Science - Emergence and Dynamics of Coherent Structures. New Yok: Oxford University Press; 2003.

20. Gillis PP, Gilman JJ. Dynamical Dislocation Theory of Crystal Plasticity. II. Easy Glide and Strain Hardening. Journal of Applied Physics. 1965;36(11):3380-3386.

21. Newnham RE. Properties of Materials - Anisotrophy, Simmetry, Structure. New York: Oxford University Press; 2005.

22. Lüthi B. Physical Acoustics in the Solid State. Berlin: Springer; 2007.

23. Nabarro FRN. Strength of Metals and Alloys. Oxford: Pergamon Press; 1986.

24. Zaiser M, Seeger A. Long-range internal stresses, dislocation patterning and work hardening in crystal plasticity. In: Nabarro FRN, ed. Dislocations in Solids. Amsterdam: North Holland; 2002. p. 1-100.

25. Messerschmidt U. Dislocation Dynamics During Plastic Deformation. Berlin: Springer; 2010.

26. Zuev LB, Semukhin BS. Some acoustic properties of a deforming medium. Philosophical Magazine A. 2002;82(6):1183-1193.

27. Murray JD. Mathematical Biology - An Introduction. New York: Springer; 2002.

28. Davydov VA, Davydov NV, Morozov VG, Stolyarov MN, Yamaguchi T. Autowaves in the moving excitable media. Condensed Matter Physics. 2004;7(3):565-578.

29. Waller I, Kapral R. Spatial and temporal structure in systems of coupled nonlinear oscillators. Physical Review A. 1984;30(4):2047.

30. Pelleg J. Mechanical Properties of Materials. Dordrecht: Springer; 2012.

31. Kocks UF, Mecking H. Physics and phenomenology of strain hardening: the FCC case. Progress in Materials Science. 2003;48(3):171-273.

32. Zuev LB. Using a crystal as a universal generator of localized plastic flow autowaves. Bulletin of the Russian Academy of Sciences: Physics. 2014;78(10):957-964.

33. Landau LD, Lifshitz EM. Fluid Mechanics. 2nd ed. Oxford: Pergamon Press; 1987.

34. V.I. Al'shits, V.L. Indenbom, Dinamika dislokatsii. In: Nabarro FRN, ed. Dislocations in Solids. Amsterdam: New Holland; 1986.

35. Kadomtsev BB. Dynamics and information. Physics-Uspekhi. 1994;37(5):425.

36. Zuev LB, Barannikova SA. Experimental study of plastic flow macro-scale localization process: Pattern, propagation rate, dispersion. International Journal of Mechanical Sciences. 2014;88:1-7.

37. Burnett JK, ed. Theory and Uses of Acoustic Emission. New York: Nova Science Publishers; 2012.

38. Caillard D, Martin JL, eds. Thermally Activated Mechanisms in Crystal Plasticity. Volume 8. Oxford: Pergamon; 2003.

39. Tokuoka T, Iwashizu Y. Acoustical birefringence of ultrasonic waves in deformed isotropic elastic materials. International Journal of Solids and Structures. 1986;4(3):383-389.

40. Landau LD, Lifshitz EM. Quantum Mechanics (Non-relativistic Theory). London: Butterworth-Heinemann; 1977. 
41. Hudson DJ. Statistics. Lectures on Elementary Statistics and Probability. Geneva: CERN; 1964.

42. Madelung O. Introduction to Solid-State Theory. Berlin: Springer Science; 1996.

43. Billingsley JP. The possible influence of the de Broglie momentum-wavelength relation on plastic strain "autowave" phenomena in "active materials". International Journal of Solids and Structures. 2001;38(24-25):4221-4234.

44. Zuev LB. The linear work hardening stage and de Broglie equation for autowaves of localized plasticity. International Journal of Solids and Structures. 2005;42(3-4):943-949.

45. Barannikova SA, Ponomareva AV, Zuev LB, Vekilov YK, Abrikosov IA. Significant correlation between macroscopic and microscopic parameters for the description of localized plastic flow auto-waves in deforming alloys. Solid State Communications. 2012;152(9):784-787.

46. Morozov EM, Fridman YB. Trajectories of the brittle-fracture cracks as a geodesic lines on the surface of a body. Soviet Physics - Doklady. 1962;6(7):619-621.

47. Olemskoi AI. Theory of Structure Transformations in NonEquilibrium Condensed Matter. New York: Nova Science Publishers; 1999.
48. Landau LD, Lifshitz EM. Statistical Physics. London: Butterworth-Heinemann; 1980.

49. Srivastava GP. The Physics of Phonons. New York: Taylor and Francis; 1990

50. Umezava H, Matsumoto H, Tachiki M. Thermo Field Dynamics and Condensed States. Amsterdam: North-Holland; 1982.

51. Friedel J. Dislocations. Oxford: Pergamon Press; 1964.

52. Kuhlmann-Wilsdorf D. The LES theory of solid plasticity. In: Nabarro FRN, Duesbery MS, eds. Dislocations in Solids. Amsterdam: New Holland; 2002. p. 211-342.

53. Kadic A, Edelen DGB. A Gauge Theory of Dislocations and Disclinations. Berlin Heidelberg: Springer-Verlag; 1983.

54. Zuev LB, Danilov VI. Plastic deformation modelled as a selfexcited wave process at the meso- and macro-level. Theoretical and Applied Frature Mechanics. 1998;30(3):175-184.

55. Zuev LB, Danilov VI. A self-excited wave model of plastic deformation in solids. Philosophical Magazine A. 1999;79(1):43-57.

56. Zuev LB, Danilov VI, Barannikova AS, Zykov IY. A new type of plastic deformation waves in solids. Applied Physics A. 2000;71(1):91-94. 